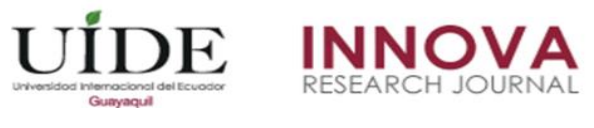

INNOVA Research Journal, ISSN 2477-9024

(Mayo, 2018). Vol. 3, No.5 pp. 99-109

DOI: https://doi.org/10.33890/innova.v3.n5.2018.533

URL: http://revistas.uide.edu.ec/index.php/innova/index

Correo: innova@uide.edu.ec

\title{
Estrategias y recursos tiflotecnológicos utilizados por docentes universitarios con estudiantes con limitaciones visuales
}

\section{Strategies and typhlotechnological resources used by university teachers with students with visual limitations}

\author{
Narlys Villalobo \\ Yaninis Machado \\ Matilde Bolaño García \\ Lucia Bustamante \\ Universidad del Magdalena-Grupo de Investigación GINFED, Colombia \\ Autor para correspondencia: narlyspatry@gmail.com, yanninys@gmail.com \\ Fecha de recepción: 25 de Octubre de 2017 - Fecha de aceptación: 15 de Mayo de 2018
}

Resumen: Se hace relevante este estudio, el cual pretende develar las estrategias y recursos tiflotecnológicos utilizados por los docentes en las clases con estudiantes con limitaciones visuales, además conocer el cumplimiento de la normativa referente a esta población estudiantil en la Universidad del Magdalena de Santa Marta - Colombia, basándonos en la ley 1680 del 2013 y algunos teóricos sobre el tema. En relación a la metodología, la investigación se orientó desde la perspectiva cualitativa, se asumió el método fenomenológico. Se basó en los aportes de Smith, M. (1998), Ministerio de Educación Nacional (2006), Cabrera \& Pesantez, (2015), Cochran- Instituto Nacional Para Ciegos. (2011), Moposita, P. (2015), Vega, M. (2011), Peña, N. (2014), Hernández, C. (2011), Piaget, J. (1983). Entre otros autores. La población estuvo conformada por 10 docentes pertenecientes a los diferentes programas académicos de la Universidad del Magdalena, que han tenido contacto con los estudiantes que poseen limitaciones visuales, con el fin de poder recolectar la información pertinente. La investigación está enfocada bajo el modelo cualitativo utilizando el diseño de la investigación acción, puesto que nos permite interpretar los diferentes acontecimientos que se presentan en la Universidad del Magdalena. Así mismo el trabajo se realiza gracias a la colaboración de los estudiantes y docentes de los diferentes programas de la Universidad del Magdalena, actores fundamentales de esta investigación. Concluyendo que los docente no conocen estrategias, ni recursos tiflotecnológicos que permita el fortalecimiento del aprendizaje. Haciéndose necesario que la Universidad del Magdalena cualifique a sus docentes, para potencializar y enriquecer el proceso de aprendizaje en los estudiantes con limitación visual Palabras Claves: recursos; estrategias; inclusión; aprendizaje; tiflotecnología; aprendizaje; adaptaciones curriculares

Abstract: This study becomes relevant, which aims to unveil the strategies and technical resources used by teachers in classes with students with visual limitations, in addition to knowing the compliance with the regulations regarding this student population in the Magdalena University of Santa Marta - Colombia, based on the law 1680 of 2013 and some theorists on the subject. In relation to the methodology, the research was oriented from the qualitative perspective, the 
phenomenological method was assumed. It was based on the contributions of Smith, M. (1998), Ministry of National Education (2006), Cabrera \& Pesantez, (2015), Cochran- National Institute for the Blind. (2011), Moposita, P. (2015), Vega, M. (2011), Peña, N. (2014), Hernández, C. (2011), Piaget, J. (1983). Among other authors. The population consisted of 10 teachers belonging to the different academic programs of the University of Magdalena, who had contact with students who have visual limitations, in order to collect relevant information. The research is focused on the qualitative model using the design of action research, since it allows us to interpret the different events that occur in the Universidad del Magdalena. Likewise, the work is carried out thanks to the collaboration of the students and teachers of the different programs of the Universidad del Magdalena, fundamental actors of this research. Concluding that the teachers do not know strategies, nor typhlotechnological resources that allow the strengthening of learning. It is necessary for the Universidad del Magdalena to qualify its teachers, to empower and enrich the learning process in students with visual limitations

Key Words: resources; strategies; inclusion; learning; tiflotechnology; learning; curricular adaptations

\section{Introducción}

Las exigencias educativas del siglo XXI requieren de innovaciones pedagógicas y una estructura de universidad donde prevalezca el respeto a la diversidad estudiantil. Este desafío enfrenta a la universidad colombiana a la creación de una enseñanza abierta al reconocimiento estudiantil desde sus diferentes aspectos, la cual permita crear un espacio para el desarrollo integral, evitando la discriminación y las desigualdades sociales.

Esta nueva visión educativa deja a un lado la educación tradicionalista que ha imperado por muchas décadas en el ámbito universitario para dar paso a una educación para todos y todas, que tenga como norte el respeto a la heterogeneidad y las oportunidades de aprendizaje desde la oferta de recursos y apoyos especializados para satisfacer las necesidades educativas del alumno.

En la actualidad la Universidad del Magdalena cuenta con una población estudiantil con limitación visual quienes poseen ritmos y estilos de aprendizaje diferentes al resto de la población. Consecuente con las nuevas políticas educativas y desde la perspectiva de una educación inclusiva es menester la creación de estrategias metodológicas y la utilización de recursos tiflotecnológicos que permitan procesos de enseñanza- aprendizaje acordes a las necesidades de esta población estudiantil y su integración al campo profesional.

Resulta evidente la importancia que enfrenta en la actualidad la Universidad del Magdalena no es en sí la limitación visual de los estudiantes, sino las estrategias metodológicas que utilizan los docentes en su práctica pedagógica que impide la incorporación de estos estudiantes a una enseñanza equitativa con el resto de la población estudiantil.

Por lo anterior, el presente estudio pretende develar las estrategias que utilizan los docentes de la Universidad del Magdalena para llevar a cabo el proceso de enseñanza aprendizaje en los estudiantes con limitaciones visuales matriculados en esta institución. En ese sentido, surge la pregunta problema ¿Cuáles son las estrategias que utilizan los docentes de la Universidad del Magdalena, para llevar a cabo sus labores académicas con estudiantes que limitaciones visuales? 


\section{Objetivo de la investigación}

Ante los anteriores planteamientos, surge el siguiente objetivo de para la formulación de una investigación. Develar las estrategias que utilizan los docentes de la Universidad del Magdalena para llevar a cabo el proceso de enseñanza - aprendizaje en los estudiantes con limitaciones visuales matriculados en esta institución.

\section{Recursos tiflotecnológicos}

Los recursos tiflotecnológicos son el resultado de la tiflotecnología, y diremos que un recurso de tal talante es aquel que facilite, apoye y brinde una comodidad al individuo ciego; es decir no solo se trata de aparatos electrónicos o informáticos, sino de utensilios como termómetros, libros braille, bastones, perros guía, directorios, cartas de restaurante etc.

La magnitud en que estos recursos influye en el desarrollo integral de las personas ciegas es tal, que hoy en día podemos encontrar en los diferentes espacios sociales (Educativos, laborales y culturales) gente que aunque padece ceguera, se incluye en toda una gama de actividades por medio de estas tecnologías; programadores WEB que trabajan por medio de lectores de pantalla, genios en telecomunicaciones que trabajan en redes de información electrónica, deportistas que ganan medallas a nivel internacional, empleados de alta productividad que con tener a su disposición una computadora común y corriente, rinden el doble de lo que empleados "normales" alcanzan, etc.

Otro ámbito importante donde estas tecnologías inciden de manera substancial en la inclusión de personas ciegas, son los espacios educativos; los lectores de pantalla, los amplificadores de imagen, los libros digitales y el braille, permiten a los alumnos ciegos estar a nivel de los jóvenes normo visuales, aprovechar la diversidad de información y medios que facilitan hoy en día el aprendizaje escolar. Es de esta guisa, que se vuelve indispensable entrenarse en el manejo de tales recursos, no solo como discapacitado, sino también, para poder generarlos y entender que hoy día las actividades que en algún momento parecieron inalcanzables para las personas invidentes, son tan reales como las que lleva a cabo una persona normo visual.

\section{Recursos tiflotecnológicos aplicados en la educación}

Al hablar de recursos tiflotecnológicos es necesario adentrarnos en las nuevas tecnologías puesto que estas no solo facilitan el funcionamiento del mundo productivo, sino que, aplicadas a la educación en general, ayudan a los procesos de aprendizaje. En Educación Especial en particular, hay que añadir un esfuerzo más para mejorar las interfaces de manera que cualquier niño o niña con discapacidad tenga la misma accesibilidad de todos para mejorar sus procesos de aprendizaje.

Por consiguiente Hernández (2011) menciona que la inquietud por educar a las personas con discapacidad visual data desde mucho antes del siglo XVIII, cuando se creó la primera escuela para niños y adolescentes ciegos en Francia, que dio inicio al desarrollo de la pedagogía especial para ciegos o tiflopedagogía. Tiflo proviene de la palabra griega Tiflus, que significa ciego. Su objeto de estudio es la educación, el proceso de enseñanza-aprendizaje de los niños y adolescentes con discapacidad visual. Este concepto se enfrenta al objetivo de desarrollar nuevas tecnologías o de adaptar y dotar de accesibilidad a las tecnologías existentes para su utilización y 
aprovechamiento por parte de las personas con discapacidad visual. Las nuevas tecnologías aplicadas a la discapacidad visual son los sintetizadores de voz, los lectores de pantalla, los lectores ópticos de caracteres, el teclado en braille y los magnificadores de texto e imagen, escáner parlante, entre otros. A través de estos aditamentos, se pueden realizar adaptaciones de materiales didácticos.

\section{Sistema de braille}

Uno de los aspectos más importantes de la educación de las personas con discapacidad visual tiene que ver con el acceso a la información, por tanto, se hace indispensable abarcar uno de los recursos como lo es el sistema de braille, puesto que permite el acceso de la información de las personas con limitación visual.

El sistema braille, inventado en el siglo XIX, está basado en un símbolo formado por 6 puntos: aquellos que estén en relieve representarán una letra o signo de la escritura en caracteres visuales.Es importante destacar que no es un idioma, sino un código. Por lo tanto, las particularidades y la sintaxis serán las mismas que para los caracteres visuales. El tamaño y distribución de los 6 puntos que forman el llamado Signo Generador, no es un capricho sino el fruto de la experiencia de Louis Braille. Las terminaciones nerviosas de la yema del dedo están capacitadas para captar este tamaño en particular.Pero este signo sólo permite 64 combinaciones de puntos, siendo insuficientes para toda la variedad de letras, símbolos y números de cada idioma.

\section{Lectores de pantallas}

Los lectores de pantallas son un tipo de ayuda técnica que permite a las personas con algún tipo de discapacidad visual el uso de ordenadores y de otros tipos de dispositivos informáticos como móviles o tabletas. Su funcionamiento se fundamenta en la transformación del texto disponible en la pantalla en voz sintetizada, de manera que las personas que los utilizan puedan escuchar el contenido con el que están interactuando. Sin lectores de pantalla, las personas ciegas o con muy poco resto de visión dependerá de terceros para realizar tareas tan cotidianas como acceder a Internet, consultar el correo electrónico o utilizar un procesador de textos, agudizando de esta manera la brecha digital entre personas con y sin discapacidad. Los lectores de pantalla también benefician a otros colectivos como personas con trastornos de lenguaje que se benefician de un refuerzo por voz en la lectura o a personas que están aprendiendo idiomas.

\section{Audiolibro}

El audiolibro es un medio de comunicación útil cuando la lectura directa no es posible. Permite conservar materiales que de otra manera podrían deteriorarse y perderse. Permite llevar a cabo otras actividades mientras se los ejecuta: conducir, caminar, tomar sol, cocinar, etc. Es fácil de descargar, de ejecutar y económico. Fomenta la lectura y promociona el contenido más que el formato escrito.

Un audiolibro puede ofrecer los contenidos más variados: puede ser un cuento, una conferencia, un curso, un artículo, un ensayo, un diálogo, una entrevista, un programa de radio, un relato más o menos largo realizado por una emisora de radio... Debido a una lógica limitación de tiempo, los audiolibros cuyo texto proviene de una obra previa de gran extensión suelen ser versiones reducidas. 


\section{Magnificador de imagen}

Un magnificador de imagen es un equipo diseñado para las personas con baja visión, con un estilo de vida activo, para que puedan acceder a la información y al goce pleno de su vida en sociedad. Está dotado de una cámara de ampliación que proyecta a una pantalla la imagen del objeto capturado. Dependiendo de la deficiencia visual de cada persona y su enfermedad específica, el magnificador de imagen permite a su usuario modificar contraste, color, agudeza, brillo y foco, de acuerdo con sus propias necesidades. Permite leer periódicos, libros, revistas, mapas, impresiones, etiquetas y ver fotografías, ilustraciones y objetos tridimensionales

\section{Magnificadores de pantalla}

Este tipo de programas simula el efecto de una lupa sobre la pantalla del computador. Está especialmente indicado para personas con deficiencia visual escasa, ya que permiten la ampliación de la pantalla completa o una parte de ella, en diferentes grados de aumento (zoom), con la finalidad de que sea más fácilmente perceptible. Los magnificadores de pantalla pueden tener determinados problemas de accesibilidad, relacionados con las configuraciones de tamaños enormes o la visualización de contenido dinámico (videos y animaciones). En el primer caso sería por la aparición de barras de desplazamiento horizontal y/o vertical, y en el segundo el magnificador intentará focalizar todo lo que se mueva y el usuario, probablemente, no pueda determinar qué ocurre en la animación o el video, pues únicamente puede visualizar una zona muy limitada de la imagen. Ejemplos de magnificadores: Magic, Zoomtext xtra, The Magnifier, Supernova, iZoom, Lunar, Bigshot

\section{Dispositivos ubicuos (Smartphone, tablet, entre otros)}

Durante la última década, el desarrollo de los Smartphones ha sido espectacular. Su gran cantidad de servicios disponibles hacen que su uso sea indispensable durante cualquier momento del día. Además, en los últimos años, los Smartphones siguen ampliando sus horizontes para captar a nuevos usuarios. Por ejemplo, el uso de un teléfono táctil por ciegos hasta hace cinco años era una utopía o una locura, sin embargo, con los avances logrados hasta la fecha y las nuevas aplicaciones disponibles, hacen que su uso pueda ser tan sencillo como para una persona sin problemas de visión. Aplicaciones como TalkBack o TextToSpeech de Android han hecho posible que personas con deficiencia visual puedan utilizar perfectamente este tipo de dispositivos.

Los Smartphones, como se ha dicho con anterioridad, están buscando aumentar su cuota de mercado mejorando su accesibilidad a personas que, debido a alguna discapacidad física, no pudieran utilizarlos correctamente con anterioridad. Gracias a este hecho, a partir de la versión 4.0 Ice Cream Sandwich de Android, la evolución estos dispositivos en accesibilidad se ha incrementado y una gran cantidad de aplicaciones, tanto de Google como de otros fabricantes, han surgido en Google Play, el mercado de aplicaciones de Android.

\section{Metodología}

La presente investigación se encuentra enfocada en el modelo cualitativo empleando un diseño de la investigación acción, puesto que nos permite realizar un diagnóstico orientado a las estrategias que utilizan los docentes de la Universidad de Magdalena en estudiantes con limitaciones visuales; así mismo, es notable que la investigación acción favorece a este proyecto, 
porque permite la interpretación de lo que ocurre desde el punto de vista de quienes actúan e interactúan en la situación problema, por ejemplo, profesores y estudiantes. Para cualquier investigación es de gran importancia la población por tanto es necesario abarcar su acepción, según Bravo (1998), citado por Arias (2012) “el universo está conformado por toda la población o conjunto de unidades que se quiere estudiar y que podrían ser observadas individualmente en el estudio".

Así mismo la población objeto de esta investigación se encuentra situada en la Universidad del Magdalena, Ubicada en la Carrera 32 N² 22- 08 San Pedro Alejandrino de Santa Marta-Magdalena la cual, se caracteriza por ser una zona estudiantil con un extracto de 3, 4, 5 medio en el cual se ve fomentado un ambiente educativo.

La muestra es la que puede determinar la problemática, ya que es capaz de generar los datos con los cuales se identifican las fallas dentro del proceso. Según Tamayo, T. Y Tamayo, M (1997), afirma que la muestra "es el grupo de individuos que se toma de la población, para estudiar un fenómeno estadístico" (p.38). El instrumento de recolección de información y el cuestionario construyeron dos cuestionarios a través de las categorías estrategias metodológicas diseñadas para personas con limitación visual y recursos didácticos, se realizó través de las dimensiones, estrategias didácticas bajo el enfoque constructivista y recursos tiflotecnológicos, con los indicadores (razonamiento lógico, formación de la personalidad, intercambio sociocultural, experimentación, motivación, sistema de braille, lectores parlantes, audiolibros, magnificadores de imagen, Dispositivos ubicuos, Escáner).

Utilizando la escala de Likert (1932), con cinco alternativas de respuesta, a saber: siempre (1), casi siempre (2), Algunas veces (3) pocas veces (4) nunca (5), el primer cuestionario posee (16) ítems y segundo (16) ítems para una sumatoria de 32 ítems. dan respuesta al segundo objetivo conocer desde las voces de los docentes las estrategias y los recursos tiflotecnológicos utilizados en las clases con estudiantes con limitaciones visuales; con el fin de poder recolectar la información deseada con dichos sujetos. Así mismo para la validación del instrumento estuvo a cargo de cuatro expertos. Identificando la relación con los ítems, el grado de pertinencia, precisión y de adecuación desde el punto de vista de su definición y formulación sintáctica al objeto de estudio.

\section{Resultados obtenidos}

Este análisis se realizó luego de la interpretación de las respuestas obtenidas por los estudiantes que hicieron parte de la población seleccionada, una vez aplicado los instrumentos, establecidos en gráfica y sistematizada, se comienza a describir los resultados del instrumento guía de observación que se relaciona con el objetivo: conocer las estrategias y recursos tiflotecnológicos utilizados en las clases con estudiantes con limitaciones visuales.

\section{¿Ha utilizado el sistema de braille en compañía de estudiante con limitaciones visuales?}




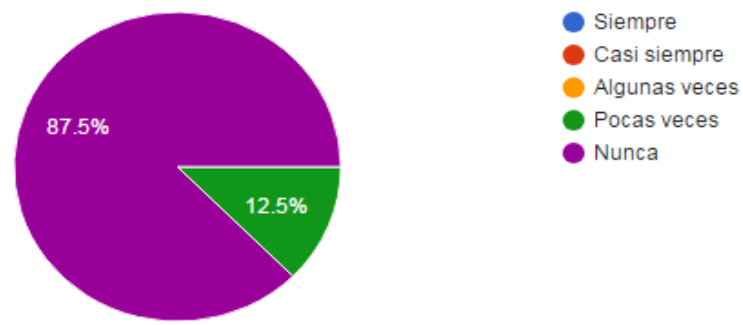

Gráfica $N^{\circ}$ 1. Sistema de braille. Utilización

Teniendo en cuenta los resultados que se presentan en la gráfica con un porcentaje de $100 \%$ se evidencia que los docentes nunca han utilizado el sistema de braille. Dado lo anterior se observa que los docentes de la Universidad del Magdalena nunca utilizan este recurso en el salón de clases, para potencializar el proceso de aprendizaje en esta población. Según Solano (2015) "el braille es el principal medio de comunicación escrito para los ciegos o personas con baja visión, esto refleja la urgencia de traducir retos en tinta a este sistema, caso contrario su derecho a la educación estará siendo vulnerado". Por tanto, se hace indispensable la utilización de este recurso en el aula de clases, con el fin de crear una mayor interacción en la comunicación y participación del docente con los estudiantes que poseen limitación visual.

\section{¿Utiliza computador con lector de pantalla para los estudiantes con limitaciones visuales?}
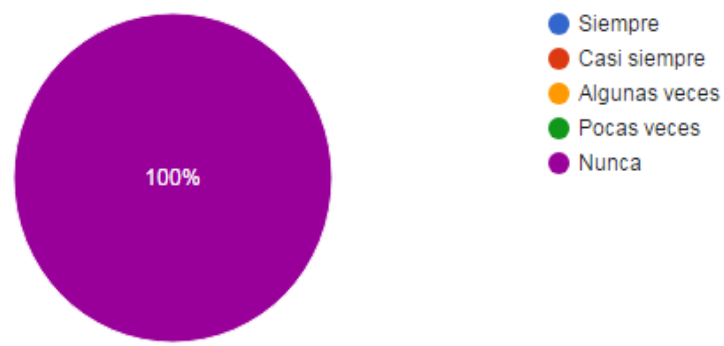

Gráfica $\mathbf{N}^{\circ}$ 2. Lectores parlantes. Utilización

De lo expuesto se colige que el $87,5 \%$ respondió que nunca han utilizado un computador con algún lector de pantalla, así mismo se evidencia que el $12,5 \%$ pocas veces utiliza dicho recurso; mostrándose así, que existen recursos que ayudan a la formación y potencialización del aprendizaje de los estudiantes con limitación visual, los docentes de la Universidad no los utilizan, puesto que desconocen la existencia de estos, de igual manera es necesario recalcar que la utilización de estos recursos permite la transformación de textos en voz sintetizadas, con la finalidad que el estudiante con limitación visual pueda leer sin ningún problema.

En su búsqueda por potencializar la investigación ¿Ha motivado a los estudiantes con limitación visual a enriquecer su aprendizaje a través de audiolibros electrónicos?
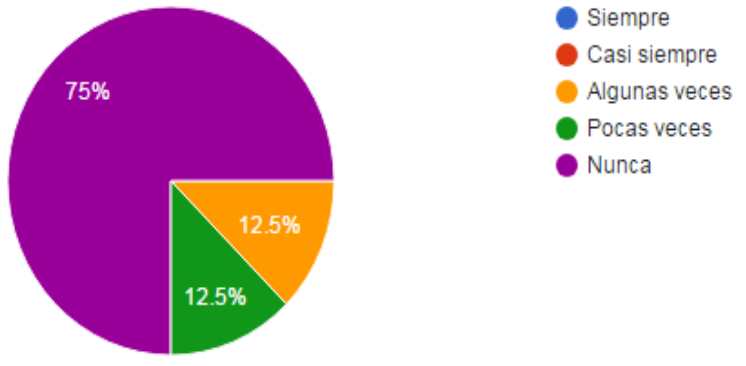


\section{Gráfica $N^{\circ}$ 3. Audiolibros.}

Se puede evidencia de la anterior gráfica que el 75\% de los docentes expresan que nunca han motivado al estudiante con limitación visual en la búsqueda de audiolibros electrónicos, por otro lado, se evidencia que coinciden los mismos porcentajes para las alternativas de respuestas "Algunas veces" y "pocas veces" 12,5\% para una totalidad de $100 \%$.

La mayoría de los docentes encuestados manifiestan que nunca han motivado al estudiante con limitación visual en utilizar este recurso como lo son los audiolibros, limitando el desarrollo del aprendizaje de los estudiantes con limitación visual, ya que este un recurso que fortalece el proceso de aprendizaje en ellos. Chacón (2007, p.261) establece cómo la incorporación de las TIC a personas con discapacidad debe verse "como una herramienta que permita el desarrollo personal, la realización de actividades y el disfrute de situaciones desde su propia individualidad, así como para su participación plena y activa en las actividades de su entorno".

\section{¿El estudiante con limitación visual emplea visores de pantalla para observar imágenes entre} otros?
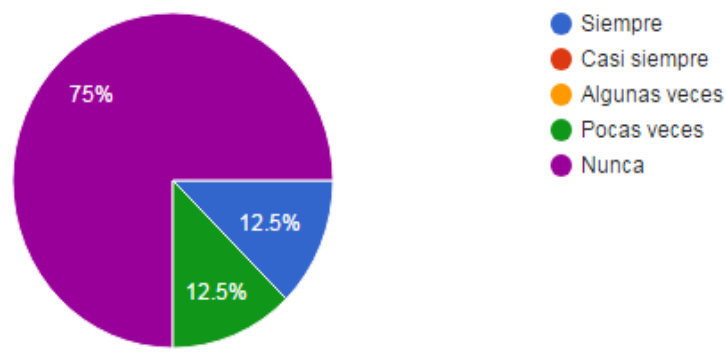

Gráfica $N^{\circ}$ 4. Magnificadores de imagen. Visores

Cómo se logra apreciar en la gráfica con las respuestas proporcionadas por los docentes el $75 \%$ de los estudiantes nunca emplea visores de pantalla en el salón de clases, en esa secuencia es posible notar que dos alternativas de respuesta "siempre" y "pocas veces" poseen el mismo porcentaje de $12.5 \%$.

La mayoría de los docentes contestaron que muy pocas veces los estudiantes utilizan este recurso y algunos no lo conocen, siendo este de gran beneficio. (Abner y Lahm, 2002) "La utilización de las TIC con los deficientes visuales también ha supuesto un gran beneficio pues ajusta las características de la imagen a las características de la visión (tamaño, colores, brillo, contraste)".

¿El estudiante con limitación visual emplea dispositivos electrónicos con algún programa que le facilite la adquisición del aprendizaje? (*) 


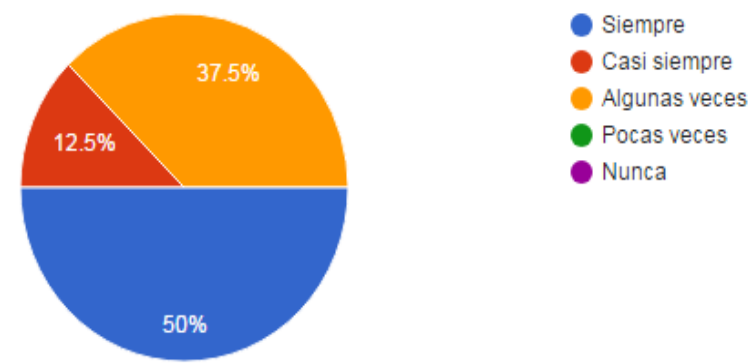

Gráfica $N^{\circ}$ 5. Dispositivos ubicuos (Smartphone, tableta, entre otros)

De lo observado en la gráfica se puede inferir que las respuestas de los docentes se han distribuido en diferentes porcentajes referente a la utilización de recursos por parte del estudiante con limitación visual, de este modo se aprecia el 50\% con la alternativa de respuesta "siempre" en esa misma secuencia algunas veces con $37.5 \%$ y el $12.5 \%$ con casi siempre para tener una totalidad del $100 \%$.

La mayoría de los docentes expresa que los estudiantes con limitación visual si emplea dispositivos electrónicos con algún programa que le facilite la adquisición del aprendizaje, ya que la tecnología ayuda a potencializar el conocimiento en estos estudiantes y favorecerlos en su diario vivir. "La importancia de las tecnologías no se encuentran en ellas mismas, sino en lo que somos capaces de realizar con ellas. Será necesario realizar un análisis para evaluar no tanto sus potencialidades tecnológicas como sus potencialidades para crear entornos educativos y comunicativos diferenciados" (Cabero, 2000).

\section{Consideraciones finales y recomendaciones}

En correspondencia con el objetivo En cumplimiento del objetivo conocer las estrategias y recursos tiflotecnológicos utilizados en las clases con estudiantes con limitaciones visuales. De acuerdo con las respuestas dadas por los docentes el $50 \%$ afirman que no conocen recursos que sirvan como apoyo para los estudiantes con limitaciones visuales, así mismo el otro 50\% responde que algunas ocasiones han notado que el estudiante utiliza su celular, grabadora y software que le permite acceder fácilmente a la información. Son muchos los autores que han analizado las posibilidades de las TIC en el medio educativo con personas con discapacidad (Negre, 2003; Cabero, Córdoba y Fernández, 2007; Hervás y Toledo, 2007; Ortega Tudela y Gómez Ariza, 2007; Prendes, 2008), citado por Muñoz (2016). Mencionan "que los usos de las TIC ayudan a superar las limitaciones que se derivan de las discapacidades cognitivas, sensoriales y motóricas del alumnado y que además favorecen la autonomía de estudiantes, pudiéndose adaptar a las necesidades y demandas de cada alumno de forma personalizada”.

Es necesario transmitir la importancia del uso de estrategias y recursos tiflotecnológicos para mejorar la enseñanza a personas con limitación visual en la Universidad del Magdalena a través de capacitaciones periódicas: sobre el uso de braille, recursos tiflotecnológicos, ya que operar bajo un mismo código de comunicación y accesibilidad la información de durante el proceso de enseñanza- aprendizaje resulta trascendental; sin embargo, si esta tarea es insostenible, la Universidad debe contar por lo menos con un traductor o monitor especializado en el tema. Así mismo los docentes requieren dominar el uso de los sistemas operativos, sea JAWS o el NVDA para lograr coordinar tareas y evaluar procesos eficazmente. 
Resulta sensato que el docente instale en su computadora y en la del estudiante un lector de pantalla, ya que los estudiantes con ceguera no pueden hacerlo por sí solos, salvo un familiar asuma esta tarea en su casa. Un ordenador sin un software lector es inutilizable para una persona con limitación visual.

Se recomienda proporcionar materiales impresos en braille y que en la biblioteca de la universidad se pueda encontrar libros, revistas, cartillas en braille, con el fin de permitirles a los estudiantes con limitación visual acceder a la información a través de dicho sistema. Es importante que previamente al iniciar el semestre los docentes realicen una entrevista o socialización con alumnos que poseen limitación visual, para conocer a profundidad su condición y así mismo realizar un diálogo a cerca de los recursos que deben utilizar o cómo puede ser más accesible la información para esta población estudiantil. De tal manera se hace indispensable trabajar con los estudiantes de la Universidad a través de charlas o capacitaciones con el fin de sensibilizar y crear un ambiente de solidaridad, responsabilidad social y ayuda mutua, sin olvidar que la familia es parte importante en el proceso de enseñanza - aprendizaje por eso necesario involucrarnos sobre todo porque jugaran el papel motivacional. Se pudo evidenciar que la universidad en su estructura física ha realizado cambios que permiten la integración de la población estudiantil con limitación visual, pero no todos los funcionarios conocen acerca de este, por tanto se recomienda realizar socializaciones que permita el conocimiento de este y otros cambios.

Propiciar actividades, planes y proyectos dentro de la universidad que permitan la integración de los estudiantes con limitación visual. Trabajar porque las acciones inclusivas en discapacidad no sean el resultado de intenciones particulares y transitorias de quienes tienen injerencia en la normatividad institucional sino que sean un asunto explícito en la política institucional y un trabajo de la comunidad académica (administrativos, docentes, estudiantes).

Por último, es importante indicar que, como todo proceso, el de la inclusión es pausado, pero el respeto a la diversidad debe ser obligatorio y la sociedad de hoy debe encaminar a una democracia inclusiva, hacia una cultura de la diversidad. Las normas, las instituciones y las políticas son legítimas para la discapacidad. La discapacidad es un asunto de todos y la inclusión de las personas con discapacidad a la educación es una alternativa a futuro para la sociedad. Una universidad incluyente será mejor en el sentido que considere las diferencias como oportunidades más no como problemas, realice un buen uso de los recursos disponibles para fortalecer el aprendizaje, utilice como punto de partida las prácticas y conocimientos existentes y desarrolle un lenguaje común dentro de la comunidad académica (Ainscow, 2001).

\section{Bibliografía}

Arias, G. (2012). El Proyecto de Investigación. Introducción a la Metodología Científica. 6ta Edición. Editorial Episteme

Ainscow, M. (2001). Comprendiendo el desarrollo de las escuelas inclusivas. Manchester: Facultad de Educación de Manchester

Abner, G.H. y Lahm, E.A. (2002). Implementation of assistive technology with students who are visually impaired: Teachers`readiness. Journal of Visual Impairment and Blindness, 96, 98105. 
Cabrera \& Pesantez, (2015). Estrategias metodológicas y recursos didácticos para el aprendizaje de estudios sociales en el octavo año de educación general básica de la sección nocturna en la unidad educativa Dolores J. Torres, periodo lectivo 2014-2015, Ecuador.

Cabero, J. (2000). Aplicación de las TICs: ¿Esnobismo o necesidad educativa? Red Digital. Revista de Tecnologías de la Información y Comunicación Educativas. Disponible en: http://reddigital.cnice.mec.es/1/firmas/firmas_cabero_ind.html (consultado el 15 de diciembre de 2011).

Cochran-Smith, M. (1998). Teacher Development and Education Reform. En A. Heargreaves y otros (eds). International Handbook of Education Change (pp. 916-951). Amsterdam: Kluwer.

Chacón, A. (2007). La atención a la diversidad con medios tecnológicos $\neg$ didácticos. En J. Ortega y A. Chacón (eds.). Nuevas tecnologías para la educación en la era digital (pp. 261 278 ). Madrid: Pirámide

Hernández, C. (2011). Desarrollo de las concepciones educativas de las personas con discapacidad visual. La Habana: Pueblo y Educación.

Ministerio de Educación Nacional (2006). Orientaciones Pedagógicas Para La Atención Educativa A Estudiantes ciegos. Recuperado de http://www.colombiaaprende.edu.co/html/micrositios/1752/articles-320691_archivo_7.pdf

Moposita, P. (2015). Estrategias metodológicas que inciden en el aprendizaje de los niños con necesidades educativas especiales de la unidad educativa Dr. Misael Acosta Solís del cantón baños, Ecuador.

Muñoz Ribón, M. (2016). Ayudas técnicas y accesibilidad web en personas con Discapacidad Visual.Nadeau, M. (2001). Juegos de relajación para niños de 5 a 12 años. España: Editorial Sirio.

Ley 1680 de 2013. Recuperado de http://www.mintic.gov.co/portal/604/articles-5006_documento.pdf

Peña, N. (2014). La diversidad en la enseñanza universitaria. España.

Solano, A (2015).Estrategias metodológicas para la inclusión educativa de personas con discapacidad visual en la universidad politécnica salesiana. Ecuador.

Tamayo y Tamayo, (1987). El Proceso de la Investigación Científica. Fundamentos de Investigación. Edit. Limusa, S.A. México D.

Vega, M. (2011). La asimilación comprende la forma en que un organismo se enfrenta a un estímulo del entorno en circunstancias actuales. Educación Internacional Bilingüe, 1-53 